\title{
On Topological Indices of Subdivided and Line Graph of Subdivided Friendship Graph
}

\author{
Hifza Iqbal*, Jabeen, Zeeshan Saleem Mufti, Muhammad Ozair Ahmad \\ Department of Mathematics and Statistics, The University of Lahore, Raiwind Road Campus, Lahore, Pakistan
}

Email address:

iqbalhifza3@gmail.com (H. Iqbal)

${ }^{*}$ Corresponding author

\section{To cite this article:}

Hifza Iqbal, Jabeen, Zeeshan Saleem Mufti, Muhammad Ozair Ahmad. On Topological Indices of Subdivided and Line Graph of Subdivided Friendship Graph. International Journal of Discrete Mathematics. Vol. 4, No. 1, 2019, pp. 57-61. doi: 10.11648/j.dmath.20190401.19

Received: February 25, 2019; Accepted: April 8, 2019; Published: May 6, 2019

\begin{abstract}
Topological indices are numerical parameters which characterizes the topology of a molecular graph, they corelate certain physo-chemical properties and importantly they are structure invariant. Degree based topological indices play vital role among others. In this paper, by means of edge dividing trick, the closed formulas of atom bond connectivity index, geometric arithmatic index, Randic index, sum connectivity index and augmented Zagreb index are computed for subdivided friendship graph and line graph of subdivided friendship graph.
\end{abstract}

Keywords: Atom Bond Connectivity Index, Geometric Arithmetic Index, Subdivided Graph, Friendship Graph

\section{Introduction}

Topological indices $[3,4,10,11,14,15,16,20,26]$ are important tools for analyzing some physicochemical properties of molecules without performing any experiment. The recently introduced Atom-Bond Connectivity index [18, 22] was defined by Ernesto Estrada [8, 9] given as follows;

$$
A B C(G)=\sum_{(u, v) \varepsilon E(G)} \sqrt{\frac{d_{u}(G)+d_{v}(G)-2}{d_{u}(G) d_{v}(G)}}
$$

There are many open problems related to $\mathrm{ABC}$ index in the mathematical chemistry literature. Interested reader can see the studies of the last two years in [1, 2, 7, 12, 23, 24]. Another recently conceived vertex-degree-based topological index is Geometric-Arithmetic index introduced by Vukiceveic and Furtula, it utilizes the difference between the geometric and arithmetic index $[5,17]$. For a simple connected graph it is defined as follows;

$$
G A(G)=\sum_{(u, v) \varepsilon E(G)} \frac{2 \sqrt{d_{u}(G) d_{v}(G)}}{d_{u}(G)+d_{v}(G)}
$$

The concept of topological indices came from Wiener, while he was working on the boiling points of paraffin and was named as the index path number. Later, it was named after him as Wiener index [21]. Hayat et al [6] studied various degree based topological indices for certain types of networks, such as Silicates, hexagonal, honeycombs and oxides. Imran et al, studied the molecular topological properties and determined the analytical closed formula for sierpinski Networks [13].

The "sum-connectivity index" is a recent invention by Bo Zhou and Nenad Trinajstic in 2009 [25, 26]. They thought that in the definition of Randic's branching index [19];

$$
R(G)=\sum_{(u, v) \varepsilon E(G)} \frac{1}{\sqrt{d_{u}(G) d_{v}(G)}}
$$

there is not a precedence reason for using the product of vertex degrees $d u \times d v$, according to them this term may be replaced by the sum $d u+d v$. The index is defined as;

$$
\operatorname{SCI}(G)=\sum_{(u, v) \varepsilon E(G)} \frac{1}{\sqrt{d_{u}(G)+d_{v}(G)}}
$$

Motivated by the success of the ABC index, Furtula put forward modified version of $\mathrm{ABC}$ index, they named it as "Augmented Zagreb index" [14, 20]. It is defined as;

$$
A Z I(G)=\sum_{(u, v) \varepsilon E(G)}\left[\frac{d_{u}(G) \cdot d_{v}(G)}{d_{u}(G)+d_{v}(G)-2}\right]^{3}
$$

The subdivision of an edge $(u, v) \varepsilon$ E means the division of edge $(u, v)$ in two edges $(u, w)$ and $(w, v)$ by introducing a new vertex $w$. The subdivision could be taken k-times where 
$\mathrm{k \varepsilon N}$. A line graph is completely a new graph, which is obtained by converting each edge of the original graph to a vertex and two vertices are adjacent if the corresponding edges have one vertex common in original graph.

\section{Method}

\subsection{Calculation of the Degree Based Topological Indices for Subdivided Friendship Graph Include the Following Procedure}

Step 1: Subdivided graph is drawn in which each edge $(\mathrm{u}, \mathrm{v})$ of the original graph is replaced by a path $\mathrm{u}-\mathrm{w}-\mathrm{v}$.

Step 2: Label each vertex of the graph by its degree.

Step 3: Count the types of degree based edges and their number, results are listed in the relevant proof.

Step 4: By using the definition of topological indices the calculations are performed and closed formulas are defined.

\subsection{Calculation of the Degree Based Topological Indices for Line Graph of Subdivided Friendship Graph Include the Following Procedure}

Step 1: The line graph of subdivided graph is obtained by replacing each edge of the subdivided graph by a vertex and two vertices are adjacent if the corresponding edges share a common vertex in the subdivided graph.

Step 2: Label each vertex of the graph by its degree.

Step 3: Count the types of degree based edges and their number, results are listed in the relevant proof.

Step 4: By using the definition of topological indices the calculations are performed and closed formulas are defined.

\section{Results and Discussion}

A friendship graph $F_{n}$, is an undirected planar graph with order $2 n+1$ and size $3 n$. The friendship graph $F n$ can be constructed by joining $\mathrm{n}$ copies of the cycle graph $C_{3}$ with a common vertex.

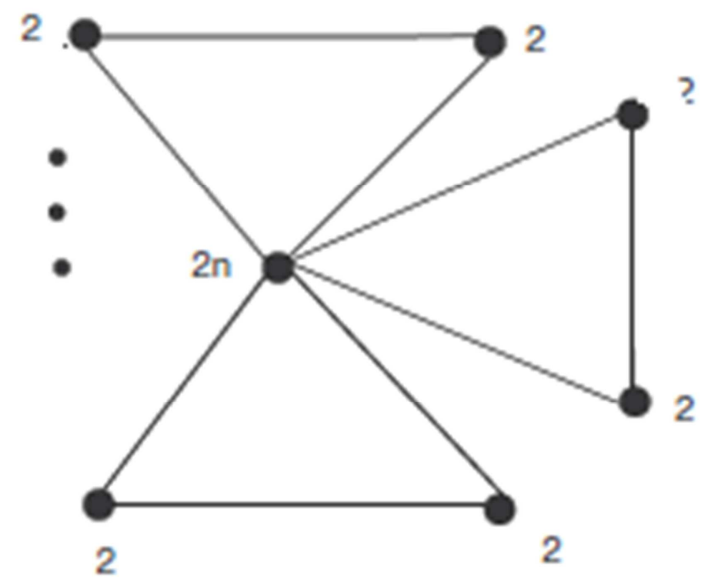

Figure 1. Friendship graph with vertex degree labelling.

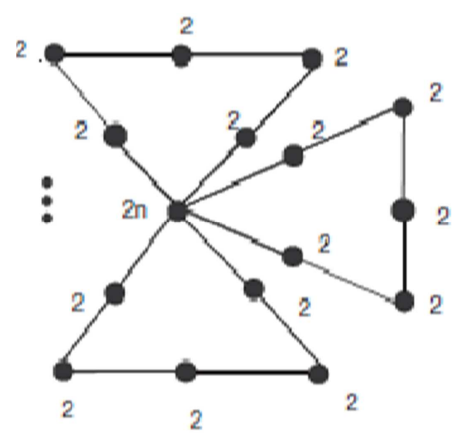

Figure 2. $k=1$, subdivided friendship graph with vertex degree labelling.

\subsection{Theorem}

Let $G_{1}$ be the graph obtained after subdividing $F_{n}$ by $k=1$. It has order $5 \mathrm{n}+1$ and size $6 \mathrm{n}$, then;
(1). $A B C\left(G_{1}\right)=6 n \sqrt{\frac{1}{2}}$
(2). $G A\left(G_{1}\right)=4 n\left(1+\frac{\sqrt{n}}{1+n}\right)$,
(3). $R\left(G_{1}\right)=2 n+\sqrt{n}$,
(4). $\operatorname{SCI}\left(G_{1}\right)=2 n\left(1+\frac{1}{\sqrt{2(1+n)}}\right)$,
(5). $\operatorname{AZI}\left(G_{1}\right)=48 n$.

Proof: For $\mathrm{k}=1$ subdivided Friendship graph has $6 n$ edges. Out of these $4 n$ edges are formed by joining vertices of degree $(2,2)$ and $2 n$ edges are formed by joining vertices of degree $(2,2 n)$.

(1). By using the information in equation 1 , we get

$$
\begin{gathered}
A B C\left(G_{1}\right)=\sum_{(u, v) \varepsilon E\left(G_{1}\right)} \sqrt{\frac{d_{u}\left(G_{1}\right)+d_{v}\left(G_{1}\right)-2}{d_{u}\left(G_{1}\right) d_{v}\left(G_{1}\right)},} \\
=4 n \sqrt{\frac{2+2-2}{2.2}}+2 n \sqrt{\frac{2+2 n-2}{2.2 n}}, \\
=4 n \sqrt{\frac{2}{4}}+2 n \sqrt{\frac{2 n}{4 n}} \\
=6 n \sqrt{\frac{1}{2} .}
\end{gathered}
$$

(2). By using the above information in equation 2, we get

$$
\begin{aligned}
G A\left(G_{1}\right) & =\sum_{(u, v) \varepsilon E\left(G_{1}\right)} \frac{2 \sqrt{d_{u}\left(G_{1}\right) d_{v}\left(G_{1}\right)}}{d_{u}\left(G_{1}\right)+d_{v}\left(G_{1}\right)} \\
& =4 n \frac{2 \sqrt{2.2}}{2+2}+2 n \frac{2 \sqrt{2.2 n}}{2+2 n} \\
& =8 n \frac{\sqrt{4}}{4}+4 n \frac{\sqrt{4 n}}{2(1+n)}
\end{aligned}
$$




$$
=4 n\left(1+\frac{\sqrt{n}}{1+n}\right) \text {. }
$$

(3). By using the above information in equation 3, we get

$$
\begin{aligned}
R\left(G_{1}\right) & =\sum_{(u, v) \varepsilon E\left(G_{1}\right)} \frac{1}{\sqrt{d_{u}\left(G_{1}\right) d_{v}\left(G_{1}\right)}} \\
= & 4 n \frac{1}{\sqrt{2.2}}+2 n \frac{1}{\sqrt{2.2 n}}, \\
= & 4 n \frac{1}{\sqrt{4}}+2 n \frac{1}{\sqrt{4 n}}, \\
& =2 n+\sqrt{n} .
\end{aligned}
$$

(4). By using the above information in equation 4 , we get

$$
\begin{aligned}
S C I\left(G_{1}\right) & =\sum_{(u, v) \varepsilon E\left(G_{1}\right)} \frac{1}{\sqrt{d_{u}\left(G_{1}\right)+d_{v}\left(G_{1}\right)}}, \\
= & 4 n \frac{1}{\sqrt{2+2}}+2 n \frac{1}{\sqrt{2+2 n}} \\
= & 4 n \frac{1}{\sqrt{4}}+2 n \frac{1}{\sqrt{2(1+n)}} \\
= & 2 n\left(1+\frac{1}{\sqrt{2(1+n)}}\right) .
\end{aligned}
$$

(5). By using the above information in equation 5, we get

$$
\begin{gathered}
A Z I\left(G_{1}\right)=\sum_{(u, v) \varepsilon E(G)}\left[\frac{d_{u}(G) \cdot d_{v}(G)}{d_{u}(G)+d_{v}(G)-2}\right]^{3}, \\
=4 n\left[\frac{2.2}{2+2-2}\right]^{3}+2 n\left[\frac{2.2 \mathrm{n}}{2+2 \mathrm{n}-2}\right]^{3}, \\
=4 n(2)^{3}+2 n(2)^{3}, \\
=48 n .
\end{gathered}
$$

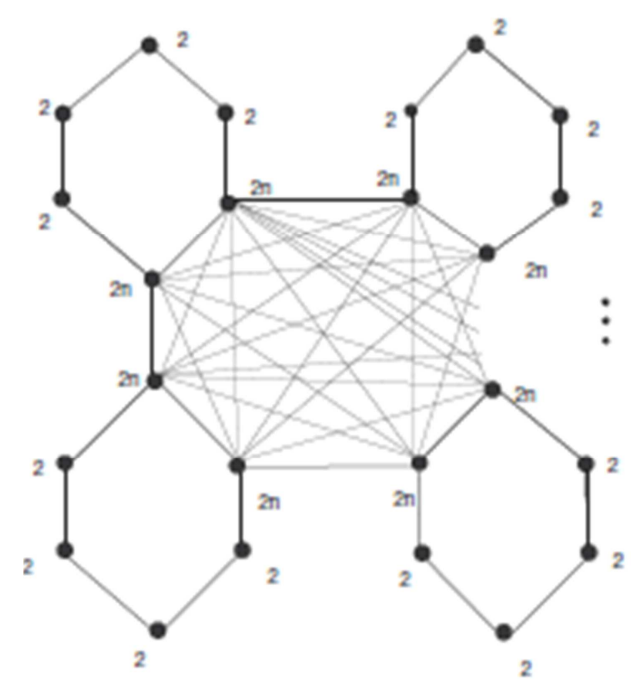

Figure 3. Line graph of $k=1$, subdivided graph with vertex degree labelling.

\subsection{Theorem}

Let $G_{2}$ be the line graph of $k=1$ subdivided friendship graph, then;
(1). $A B C\left(G_{2}\right)=\frac{5 n+(2 n-1)^{\frac{3}{2}}}{\sqrt{2}}$,
(2). $G A\left(G_{2}\right)=2 n(n+1)+\frac{4 n^{\frac{3}{2}}}{n+1}$,
(3). $R\left(G_{2}\right)=\frac{5 n}{2}+\sqrt{n}-\frac{1}{2}$,
(4). $S C I\left(G_{2}\right)=\left(\frac{3}{2}+\frac{\sqrt{2}}{\sqrt{1+n}}+\frac{2 n-1}{2 \sqrt{n}}\right) n$,
(5). $\operatorname{AZI}\left(G_{2}\right)=40 n+\frac{8 n^{7}}{(2 n-1)^{2}}$.

Proof: In $G_{2}, 3 n$ edges are formed by joining vertices of degree $(2,2), 2 n$ edge are formed by joining vertices of degree $(2,2 n)$ and $2 n^{2}-n$ edges are formed by joining vertices of degree $(2 n, 2 n)$.

(1). Using the information in equation 1 , we get

$$
\begin{gathered}
A B C\left(G_{2}\right)=\sum_{(u, v) \varepsilon E\left(G_{2}\right)} \sqrt{\frac{d_{u}\left(G_{2}\right)+d_{v}\left(G_{2}\right)-2}{d_{u}\left(G_{2}\right) d_{v}\left(G_{2}\right)}}, \\
=3 n \sqrt{\frac{2+2-2}{2.2}}+2 n \sqrt{\frac{2+2 n-2}{2.2 n}}+\left(2 n^{2}\right. \\
=3 n \sqrt{\frac{1}{2}+2 n \sqrt{\frac{2 n+2 n-2}{2}+\left(2 n^{2}-n\right)}}, \sqrt{\frac{4 n-2}{4 n^{2}}} \\
=5 n \sqrt{\frac{1}{2}+\frac{n(2 n-1) \sqrt{2(n-1)}}{2 n}} \\
=\frac{5 n+(2 n-1)^{\frac{3}{2}}}{\sqrt{2}} .
\end{gathered}
$$

(2). By using the above information in equation 2 , we get

$$
\begin{gathered}
G A\left(G_{2}\right)=\sum_{(u, v) \varepsilon E\left(G_{2}\right)} \frac{2 \sqrt{d_{u}\left(G_{2}\right) d_{v}\left(G_{2}\right)}}{d_{u}\left(G_{2}\right)+d_{v}\left(G_{2}\right)} \\
=3 n \frac{2 \sqrt{2.2}}{2+2}+2 n \frac{2 \sqrt{2.2 n}}{2+2 n}++\left(2 n^{2}-n\right) \frac{2 \sqrt{2 n .2 n}}{2 n+2 n} \\
=3 n+4 n \frac{\sqrt{n}}{(1+n)}+2 n^{2}-n, \\
=2 n(n+1)+\frac{4 n^{\frac{3}{2}}}{n+1} .
\end{gathered}
$$

(3). By using the above information in equation 3 , we get

$$
R\left(G_{2}\right)=\sum_{(u, v) \varepsilon E\left(G_{2}\right)} \frac{1}{\sqrt{d_{u}\left(G_{2}\right) d_{v}\left(G_{2}\right)}}
$$




$$
\begin{gathered}
=3 n \frac{1}{\sqrt{2.2}}+2 n \frac{1}{\sqrt{2.2 n}}+\left(2 n^{2}-n\right) \frac{1}{\sqrt{2 n \cdot 2 n}} \\
=\frac{3 n}{2}+\frac{n}{\sqrt{n}}+\frac{2 n^{2}-n}{2 n}, \\
=\frac{5 n}{2}+\sqrt{n}-\frac{1}{2} .
\end{gathered}
$$

(4). By using the above information in equation 4 , we get

$$
\begin{gathered}
S C I\left(G_{1}\right)=\sum_{(u, v) \varepsilon E\left(G_{1}\right)} \frac{1}{\sqrt{d_{u}\left(G_{1}\right)+d_{v}\left(G_{1}\right)}} \\
=3 n \frac{1}{\sqrt{2+2}}+2 n \frac{1}{\sqrt{2+2 n}}+\left(2 n^{2}-n\right) \frac{1}{\sqrt{2 n+2 n}} \\
=\frac{3 n}{2}+\frac{2 n}{\sqrt{2(1+n)}}+\frac{2 n^{2}-n}{2 \sqrt{n}} \\
=\left(\frac{3}{2}+\frac{\sqrt{2}}{\sqrt{1+n}}+\frac{2 n-1}{2 \sqrt{n}}\right) n .
\end{gathered}
$$

(5). By using the above information in equation 5 , we get

$$
\begin{gathered}
A Z I\left(G_{1}\right)=\sum_{(u, v) \varepsilon E(G)}\left[\frac{d_{u}(G) \cdot d_{v}(G)}{d_{u}(G)+d_{v}(G)-2}\right]^{3}, \\
=3 n\left[\frac{2.2}{2+2-2}\right]^{3}+2 n\left[\frac{2.2 \mathrm{n}}{2+2 \mathrm{n}-2}\right]^{3} \\
+\left(2 n^{2}-n\right)\left[\frac{2 \mathrm{n} \cdot 2 \mathrm{n}}{2 \mathrm{n}+2 \mathrm{n}-2}\right]^{3}, \\
=3 n(2)^{3}+2 n(2)^{3}+\left(2 n^{2}-n\right)\left[\frac{4 n^{2}}{4 \mathrm{n}-2}\right]^{3}, \\
=40 n+\frac{8 n^{7}}{(2 n-1)^{2}} .
\end{gathered}
$$

\section{Conclusion}

In this paper, by means of graph structure analysis, certain degree-based topological indices namely atom bond connectivity index, geometric arithmatic index, Randic index, sum connectivity index and augmented Zagreb index for $\mathrm{k}=1$ subdivided friendship graph and line graph of $\mathrm{k}=1$ subdivided friendship graph are defined. In future, some other topological indices for these structures can be calculated.

\section{References}

[1] M. S. Abdelgader, C. Wang and S. A. Mohammed (2018), Computation of Topological indices of some special graphs. mathematics.

[2] A. R. Ashrafi, Z. T. Dehghan, N. Habibi (2015), Extremal atom-bond connectivity index of cactus graphs. Commun. Korean Math. Soc. 30, 283-295.
[3] M. Baca, J. Horvathova, M. Mokrisova, A. S. Fenovcikova, A. Suhanyiova (2015), On topological indices of Carbon nanotube network, Canadian Journal of Chemistry. 93(10), 1-4.

[4] M. Baca, J. Horvathova, M. Mokrisova, A. Suhanyiova (2015), On topological indices of fullerenes, Applied Mathematics and computation 251, 154-161.

[5] K. C. Das (2010), On geometric-arithmetic index of graphs, MATCH Commun. Math. Comput. Chem. 64, 619.

[6] M. Deza, P. W Fowler, A. Rassat, K. M. Rogers, (2000), Fullerenes as tilings of surfaces. J. Chem. Inf. comput. Sci. 40, $550-558$.

[7] D. Dimitrov (2016), On structural properties of trees with minimal atom-bond connectivity index II: Bounds on and branches. Discrete Appl. Math. 204, 90-116.

[8] E. Estrada, L. Torres, L. Rodríguez, I. Gutman (1998), An Atom-Bond Connectivity Index: Modelling the Enthalpy of Formation of Alkanes, Indian Journal of Chemistry, 37A, 849-855.

[9] E. Estrada (2008), Atom-bond connectivity and the energetic of branched alkanes, Chem. Phys. Lett. 463, 422-425.

[10] M. R. Farahani, R. Kanna, W. Gao (2015) The Schultz, modified Schultz indices and their polynomials of the Jahangir graphs Jn, $\mathrm{m}$ for integer numbers $n=3, m \geq 3$. Asian J Appl Sci. 3(6): 823-827.

[11] M. R. Farahani, S. Zafar, M. N. Husin, Z. Zahid, "On the Topological Indices of the Line Graphs of Certain Pantacenic Nanostructures" 345.

[12] B. Furtula (2016), Atom-bond connectivity index versus Graovac Ghorbani analog. MATCH Commun. Math. Comput. Chem. 75, 233-242.

[13] S. Hayat, M. Imaran (2014) Computation of T.^\$opological indices of certain networks. Appl. Math. comput.240, 213-228.

[14] Y. Huang, B. Liu, and L. Gan (2012), MATCH Commun. Math. Comput. Chem. 67, Augmented Zagreb Index of Connected Graphs, 483.

[15] M. Imran, S. Akhter, W. Ga and M. R. Farahani (2018), On topological indices of honeycomb networks and Graphene.

[16] M. Imran, W. Gao, M. R. Farahani (2017) On Topological properties of Sierpinski networks. Chaos solitons Fractals, 98, 199-204.

[17] J. M. Rodzgueza , J. M. Sigarretab (2015), On the Geometric-Arithmetic Index, MATCH Commun. Math. Comput. Chem. 74, 103-120.

[18] H. Palacios (2014), A resistive upper bound for the ABC index MATCH common Math. Comput. Chem. 72, 709-713.

[19] M. Randic (1975), Characterization of molecular branching, J. Am. Chem. Soc. 97, 6609.

[20] D. Wang, Y. Huang, and B. Liu (2012), Testing the quality of molecular structure descriptors. Vertex-degree-based topological indices, MATCH Commun. Math. Comput. Chem. 68, 209.

[21] H. Wiener (1947), Structural determination of Paraffin boiing points. J. Am. Chem. Soc. 69, 17-20. 
[22] R. Xing, B. Zhou. Du, (2010) Further results on atom bond connectivity index of trees, discrete applied math 158, $1536-1545$.

[23] X. M. Zhang, Y. Yang, H. Wang, X. D. Zhang (2016), Maximum atom-bond connectivity index with given graph parameters. Discrete Appl. Math. 215, 208-217.

[24] L. Zhong, Q. Cui (2015), On a relation between the atom-bond connectivity and the first geometric arithmetic indices. Discrete
Appl. Math. 185, 249-253.

[25] B. Zhou, N. Trinajstic (2009), J. Math. Chem. On a novel connectivity index. 46, 1252.

[26] B. Zhou (2006), Bounds for the Schultz molecular topological index, MATCH Commun Math Comput Chem. 56, 189-194. 